\title{
CUIDADOS PALIATIVOS A PACIENTES COM FERIDAS ONCOLÓGICAS EM HOSPITAL UNIVERSITÁRIO: RELATO DE EXPERIÊNCIA
}

Maria Cristina Freitas de Castro', Pamella da Silva Cruz ${ }^{2}$, Marianne dos Santos Grellmann ${ }^{3}$, Willian Alves dos Santos 3 , Patricia dos Santos Claro Fuly ${ }^{4}$

${ }^{1}$ Enfermeira. Mestranda em Enfermagem Assistencial. Universidade Federal Fluminense. Niterói-RJ-Brasil.

${ }^{2}$ Enfermeira. Residente em Oncologia. Universidade Federal Fluminense. Niterói-RJ-Brasil.

${ }^{3}$ Enfermeiro. Mestrando em Ciências do Cuidado em Saúde. Universidade Federal Fluminense. Niterói-RJ-Brasil.

${ }^{4}$ Enfermeira. Doutora em Enfermagem. Universidade Federal Fluminense. Niterói-RJ-Brasil.

RESUMO: Este estudo tem como objetivo descrever o cuidado desenvolvido no ambulatório de cuidados paliativos, ao paciente portador de ferida oncológica em hospital universitário federal do município de Niterói/RJ, habilitado como Unidade de Alta Complexidade em Oncologia. Foi desenvolvido por meio da observação das ações de enfermagem na implementação da Política Nacional para Prevenção e Controle do Câncer. A complexidade do cuidado às feridas oncológicas requer competência técnica e relacional com abordagem às dimensões física, psicológica, social e espiritual, essenciais para a qualidade de vida do paciente e família. O ambulatório iniciou suas atividades em 2005, tendo em outubro de 2014 uma média de 70 pacientes em atendimento e entrada de 12 pacientes novos ao mês. Reconhecer os desafios inerentes às tomadas de decisão, nesta proposta de cuidado frente às demandas do paciente e prover um suporte adequado ao paciente e cuidador são aspectos importantes para um cuidado eficaz. DESCRITORES: Cuidados paliativos; Enfermagem oncológica; Ferimentos e Lesões.

\section{PALLIATIVE CARE FOR PATIENTS WITH ONCO- LOGICAL WOUNDS IN A TEACHING HOSPITAL: AN EXPERIENCE REPORT}

\begin{abstract}
This study aimed to describe the care provided in the palliative care outpatient center, for the patient with an oncological wound in a federal university teaching hospital in the municipality of Niterói in the state of Rio de Janeiro (RJ), certified as a High Complexity Oncology Center. It was undertaken through observation of the nursing actions in the implementation of the National Cancer Prevention and Control Policy. The complexity of the care for the oncological wounds requires technical and relational competence, addressing the physical, psychological, social and spiritual dimensions, essential for the quality of life of the patient and their family. The outpatient center began its activities in 2005; in October 2014 it had a mean of 70 patients being attended, with 12 new patients entering each month. Recognizing the challenges inherent to decision taking in this proposal of care regarding the needs of the patient, and providing appropriate support for the patient and caregiver, are important aspects if one is to provide efficacious care.

DESCRIPTORS: Palliative care; Oncology nursing; Wounds and Injuries.
\end{abstract}

\section{CUIDADOS PALIATIVOS A PACIENTES CON HERIDAS ONCOLÓGICAS EN HOSPITAL UNIVERSITARIO: RELATO DE EXPERIENCIA}

RESUMEN: Este estudio tiene como finalidad describir el cuidado desarrollado en el ambulatorio de cuidados paliativos, al paciente con herida oncológica en hospital universitario federal del municipio de Niterói/RJ, habilitado como Unidad de Alta Complejidad en Oncología. Fue desarrollado por medio de observación de las acciones de enfermería en la implementación de la Política Nacional para Prevención y Control del Cáncer. La complejidad del cuidado a las heridas oncológicas necesita competencia técnica y relacional con abordaje a las dimensiones física, psicológica, social y espiritual, fundamentales para la cualidad de vida del paciente y de la familia. El ambulatorio empezó sus actividades en 2005, habendo en octubre de 2014 una media de 70 pacientes en atendimiento y aceptación de 12 pacientes nuevos al mes. Es esencial para un cuidado eficaz reconocer los desafíos inherentes a cada decisión, en esta propuesta de cuidado delante de las demandas del paciente, y dar soporte adecuado al paciente y cuidador.

DESCRIPTORES: Cuidados paliativos; Enfermería oncológica; Heridas y lesiones. 


\section{INTRODUÇÃO}

Estimativas do Instituto Nacional de Câncer (INCA) apontam o surgimento aproximado de 576 mil novos casos de câncer em 2014/2015(1). Frente a este quadro, o câncer passa a ser entendido como problema de saúde pública, o que aponta para a necessidade de criação de estratégias para implementação da Política Nacional para a Prevenção e Controle do Câncer, instituida em maio de $2013^{(1-2)}$. O princípio do cuidado integral organizado, com ações e serviços voltados para o cuidado da pessoa com câncer, que embasa esta política, contempla a prevenção, a detecção precoce, o diagnóstico, o tratamento e os cuidados paliativos ${ }^{(2)}$.

O Hospital Universitário em questão está cadastrado como uma Unidade de Assistência de Alta Complexidade em Oncologia (UNACON), desde 2009, estando assim habilitado para oferecer assistência especializada e integral para o diagnóstico definitivo, tratamento e acompanhamento dos cânceres mais prevalentes, com o fornecimento de medicações específicas para o tratamento e controle dos sintomas ${ }^{(3)}$.

De acordo com a Organização Mundial de Saúde (OMS), grande número dentre estes novos casos de câncer, já estará em estágio avançado quando assistidos pela primeira vez por um profissional de saúde, sendo a única opção realística de tratamento o alívio da dor e cuidados paliativos. Esta modalidade de cuidado é entendida como assistência que visa a melhoria da qualidade de vida do paciente e seus familiares, diante de uma doença que ameace a vida, por meio do tratamento da dor e demais sintomas físicos, sociais, psicológicos e espirituais ${ }^{(4)}$.

De $5 \%$ a $10 \%$ dos pacientes com câncer avançado irão desenvolver feridas oncológicas durante os seis últimos meses de vida, em decorrência do tumor primário ou metastáticos ${ }^{(5)}$. Neste contexto, este estudo tem como objetivo descrever o cuidado de enfermagem desenvolvido no ambulatório de cuidados paliativos, ao paciente portador de ferida oncológica, em um hospital universitário federal do município de Niterói/RJ.

\section{CONTEXTUALIZANDO O CUIDADO}

O ambulatório começa a funcionar em 2005, com atendimento aos pacientes encaminhados pelo serviço de onco-hematologia do hospital. Os cânceres de mama, pulmão, cólon, reto, próstata e colo de útero estão entre os prevalentes no ambulatório, tendo atualmente uma média de 70 pacientes em atendimento com entrada de 12 pacientes novos por mês. A grande maioria dos pacientes apresenta doença avançada, com uma sobrevida média de 69 dias, e não consegue prover seu autocuidado, necessitando de intervenções precisas da equipe para o controle dos sintomas angustiantes, tanto de ordem física quanto psicológica, social e espiritual, assim como capacitação dos cuidadores (familiares ou não) para a continuidade dos cuidados em casa.

Dentre as atividades desenvolvidas pela equipe de enfermagem do ambulatório, destacase o cuidado com as feridas oncológicas. Cuidado complexo e que requer uma abordagem sistematizada e integral, considerando que os problemas enfrentados pelo paciente e seus familiares envolvem múltiplas dimensões. Neste sentido, a interdisciplinaridade, inerente à equipe, é um importante aspecto nesta abordagem e ferramenta imprescindível na filosofia dos cuidados paliativos ${ }^{(5-6)}$.

As feridas oncológicas normalmente se desenvolvem durante os seis últimos meses de vida, em decorrência do tumor primário ou metastático. Como resultado da infiltração de células cancerosas nas estruturas da pele ocorre a formação de uma ferida, evolutivamente exofítica, com proliferação celular descontrolada ${ }^{(5)}$.

As localizações mais freqüentes das feridas, de acordo com os cânceres prevalentes, são mama, cabeça e pescoço, tórax anterior, genitália, períneo, virilha, costas e extremidades. Dentre os sintomas prevalentes destacam-se dor, exsudato, odor, sangramento e necrose. Entre as questões psicossociais a mudança na imagem corporal, o isolamento social, constrangimento e vergonha estão associados com o viver e cuidar de uma ferida desta natureza ${ }^{(7-10)}$.

O potencial ruim de cicatrização inerente a estas feridas, além da possibilidade de recidiva tumoral, como conseqüência da indução de divisão celular para fins de reparação tecidual, durante o uso de produtos cicatrizantes, torna importante a compreensão das metas dos cuidados paliativos, como essencial no desenvolvimento de um plano de tratamento com foco sobre o controle de sintomas e a manutenção 
ou melhora da qualidade de vida do paciente e familiar, conforto e dignidade ${ }^{(5,8)}$.

Diante desta complexidade, fica evidente a importância da consulta de enfermagem como estratégia que possibilita o estabelecimento de uma relação mais próxima com o paciente e sua família, importante unidade do cuidado, detecção precoce de sintomas com elaboração de um plano de cuidado individualizado e adequado a cada paciente atendido, com posteriores reavaliações das ações ${ }^{(11)}$. A partir de 2009, com a inclusão da enfermeira na equipe multiprofissional, iniciam-se as consultas de enfermagem.

Quando o paciente é admitido no ambulatório, ao ser identificada a presença de uma ferida oncológica, é feita uma minuciosa avaliação da ferida e do paciente, sendo formulado um plano de cuidados. A localização da ferida, aparência, tamanho, quantidade e características do exsudato, dor, odor, presença de infecção e tecido desvitalizado no leito da ferida, além do aspecto da pele ao redor da lesão são aspectos avaliados e irão guiar a terapia local. A descrição da ferida deve ser documentada de forma concisa e sistemática para assegurar credibilidade nas avaliações posteriores ${ }^{(12)}$.

Como parte desta primeira avaliação, procuramos identificar o cuidador (pessoa mais próxima, responsável pelo cuidado) e a rede de atenção básica na qual o paciente é vinculado para uma parceria no cuidado. Realizar os curativos em domicílio é considerado uma das principais dificuldades encontradas pelos cuidadores, o que implica na necessidade de capacitação deste familiar/cuidador pelo enfermeiro ${ }^{(13)}$.

Desta forma, a primeira troca do curativo é feita com o cuidador, procurando identificar suas limitações e valorizando a sua presença e importância no cuidado. A comunicação eficaz com membros da família é fundamental para o cuidado do paciente em casa. Mantê-los informados sobre os procedimentos e envolvêlos no cuidado é importante para o processo e isto pode incluir capacitá-los com habilidades necessárias para o cuidado ${ }^{(14)}$.

Ao ser liberado para casa, oferecemos um formulário elaborado pela enfermeira do setor, no qual constam orientações básicas para controle dos sintomas prevalentes e disponibilizamos contato telefônico para esclarecimento de eventuais dúvidas. Estudos indicam que pacientes e cuidadores estão despreparados para a tarefa de cuidar deste tipo de ferida ${ }^{(7,15)}$. Dessa forma, questões práticas relacionadas ao cuidado com a ferida, assim como questões emocionais são abordadas de uma maneira holística e centrada no paciente e cuidador. Enfim, todas as ações possíveis são realizadas no sentido de melhorar o suporte aos cuidadores, que muitas vezes cuidam de pessoas queridas e membros da família.

\section{CONSIDERAÇÕES FINAIS}

A progressão da doença, o aumento da ferida oncológica e a dificuldade no controle dos sintomas prevalentes podem trazer sentimentos de impotência e desânimo para o enfermeiro. O conhecimento científico dos princípios dos cuidados paliativos, com abordagem às dimensões física, psicológica, social e espiritual é essencial neste processo, assim como a compreensão da importância de uma equipe interdisciplinar, o que pode ajudar na busca de estratégias de enfrentamento da doença.

Baixo nível de informação e ausência de ajuda profissional pode levar alguns pacientes a usarem estratégias que podem exacerbar os sintomas, por outro lado o impacto que um cuidado apropriado pode ter na sensação de bem estar físico e emocional do paciente é evidente. O controle de sintomas não é somente importante para a saúde física, melhora a autoestima do indivíduo, restaura sua dignidade e qualidade de vida.

Neste contexto, cabe ao enfermeiro levantar as demandas de cuidado do paciente e família, planejar e implementar um plano de cuidados individualizado, pautado por princípios científicos e conhecimentos de enfermagem específicos, que atenda a estas demandas e proceder à avaliações freqüentes que possibilitem novos planejamentos.

A complexidade da situação pode exigir avaliações rápidas e ações precisas para o gerenciamento do cuidado, o que requer pensamento crítico que antecipe as situações de acordo com as diferentes fases da doença. Isto determina além de competência técnica a competência relacional, que inclui habilidade de escutar e se comunicar com o paciente, familiares e demais membros da equipe interdisciplinar. Também, oferece oportunidade para que os 
doentes e familiares expressem suas necessidades e sejam referenciados para profissionais especializados, se for o caso.

\section{REFERÊNCIAS}

1. Ministério da Saúde (BR). Instituto Nacional de Câncer (INCA). Estimativa 2014: incidência de câncer no Brasil. [Internet]. Rio de Janeiro: INCA, 2014 [acesso em 25 abr 2014]. Disponível: http://www.inca.gov.br/ estimativa/2014/

2. Ministério da Saúde (BR). Portaria n. 874/GM de 16 de maio de 2013. Institui a Política Nacional para a Prevenção e Controle do Câncer na Rede de Atenção à Saúde das Pessoas com Doenças Crônicas no âmbito do Sistema Único de Saúde (SUS). [Internet]. Brasília: 2013[acesso em 25 abr 2014]. Disponível: http://bvsms.saude.gov.br/bvs/saudelegis/gm/2013/ prt0874_16_05_2013.html

3. Ministério da Saúde (BR). Portaria n. 741 de 19 de dezembro de 2005. Define as Unidades de Assistência da Alta Complexidade em Oncologia (UNACON), os Centros de Assistência em Alta Complexidade em Oncologia (CACON) e os Centros de Referência em Alta Complexidade Oncológica. [Internet]. Brasília; 2005[acesso em 20 abr. 2014]. Disponível: http://bvsms.saude.gov.br/bvs/saudelegis/sas/2005/ prt0741_19_12_2005.html

4. World Health Organization [homepage na internet]. Geneva [acessado em 20 abr 2014]. Palliative care [acesso em 08 mai 2013]. Disponível: http://www.who. int/cancer/palliative/en/

5. Firmino F. Pacientes portadores de feridas neoplásicas em serviços de cuidados paliativos: contribuições para a elaboração de protocolos de intervenções de enfermagem. Rev. bras. cancerol. 2005; 51(4): 347-59.

6. Ministério da Saúde (BR). Instituto Nacional de Câncer (INCA). Ações de enfermagem para o controle do câncer uma proposta de integração ensino-serviço. $3^{\mathrm{a}}$ ed. Rio de Janeiro: INCA; 2008.

7. Probst S, Arber A, Faithfull S. Malignant fungating wounds: the meaning of living in an unbounded body. Eur J Oncol Nurs 2013; 17(1): 38-45.

8. Maida V, Ennis M, Kuziemsky C, Trozzolo L. Symptoms associated with malignant wounds: a prospective case series. J Pain Symptom Manage. 2009;37(2):206-11.

9. Woo KY, Sibbald RG. Local wound care for malignant and palliative wounds. Adv. wound care. 2010;23(9):417-28.

10. Alexander S. Malignant fungating wounds: key symptoms and psychsocial issues. J Wound Care 2009;
18(8): 325-29.

11. Rosa LM, Mercês NNA, Marcelino SR, Radünz V. A consulta de enfermagem no cuidado à pessoa com câncer: contextualizando uma realidade. Cogitare enferm. 2007; 12(4): 487-93.

12. Gerlach MA. Wound Care Issues in the Patient with Cancer. Nurs Clin North Am 2005; 40(2): 295-323.

13. Camarão RR. Cuidados com Feridas e Curativos. In: Carvalho RT, Parson HA, organizadores. Manual de Cuidados Paliativos. Rio de Janeiro: Academia Nacional de Cui-dados Paliativos; 2009. p. 306-318.

14. Wilson V. Assessment and management of fungating wounds: a review. Br J Community Nurs 2005; 10(3): 28-34.

15. Probst S, Arber A, Trojan A, Faithfull S. Caring for a loved one with a malignant fungating wound. Support Care Cancer 2012; 20(12): 3065-70. 